> La recherche bibliographique représente, en quelque sorte, une des fondations de l'édifice des connaissances sur lesquelles les chercheurs s'appuient au quotidien dans leurs laboratoires pour réaliser leurs travaux de recherche. Parmi les nombreuses bases de données bibliographiques disponibles dans le domaine biomédical (Embase, Current Contents, Biosis...), Medline PubMed est de loin la plus utilisée en raison de la gratuité de son accès. Cependant, le bon usage des différentes fonctionnalités proposées par cette base (utilisation du vocabulaire contrôlé $\mathrm{MeSH}$, réalisation de revues de sommaire, systématisation de recherches bibliographiques...), préalable indispensable à une recherche bibliographique de qualité, nécessite de posséder un certain nombre de notions qui sont exposées dans le présent article. <

\section{Recherche \\ bibliographique \\ en biologie \\ et en médecine \\ Du bon usage \\ de Medline PubMed}

Christophe Boudry, Gérard Bozet

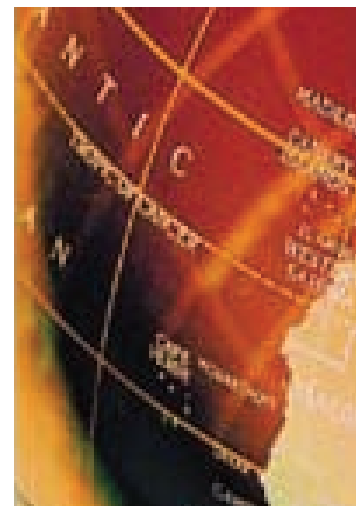

La page d'accueil de PubMed comporte trois barres de commandes ou menus (Figure 1). La barre de menu de couleur noire située au-dessus de la fenêtre d'interrogation permet de consulter d'autres bases de données (principalement factuelles). La barre de commandes située sous la fenêtre d'interrogation donne accès à des fonctionnalités qui permettent notamment de limiter la portée de ses recherches («Limits») ou d'en afficher l'historique («History»). La barre latérale de couleur bleue, à gauche de l'écran, donne accès à l'aide de recherche et à différentes interfaces très utiles aux chercheurs dans le domaine biomédical. L'objectif de cet article est de présenter ces différentes interfaces et d'en détailler le fonctionnement.

\section{Notions indispensables}

\section{Formuler correctement sa demande}

\section{à l'aide de termes MeSH}

Pour limiter les problèmes liés à l'existence de synonymes décrivant un même concept, Medline PubMed met à la disposition de ses utilisateurs le thésaurus MeSH (medical subject headings). Ce dernier est organisé selon une arborescence partant de notions générales 
pour aller vers des éléments de plus en plus spécifiques. Son intérêt est de permettre l'utilisation d'un terme unique pour décrire un concept lors de l'indexation dans Medline, alors que les auteurs peuvent employer des synonymes (par exemple: apoptosis, apoptoses, programmed cell death...). L'utilisation du thésaurus MeSH est donc fortement conseillée lors de l'interrogation de Medline PubMed, car elle permet de s'affranchir en grande partie des problèmes de synonymie et de gagner en précision. L'outil de Medline PubMed pour identifier ces termes $\mathrm{MeSH}$ est le «MeSH database ».

\section{Le MeSH database}

Cette interface est accessible en cliquant sur le lien de la barre latérale de couleur bleue, à gauche de l'écran (Figure 1). Lorsque l'utilisateur saisit un mot dans cette interface, une liste de termes lui est proposée. Dans cette liste, apparaissent les termes MeSH qui contiennent non seulement le mot saisi, mais également les termes MeSH connus pour être des synonymes de ce mot. Ainsi, la saisie des mots «programmed cell death» permet de retrouver le terme $\mathrm{MeSH}$ « apoptosis » correspondant. Dans tous les cas, l'interface propose une courte

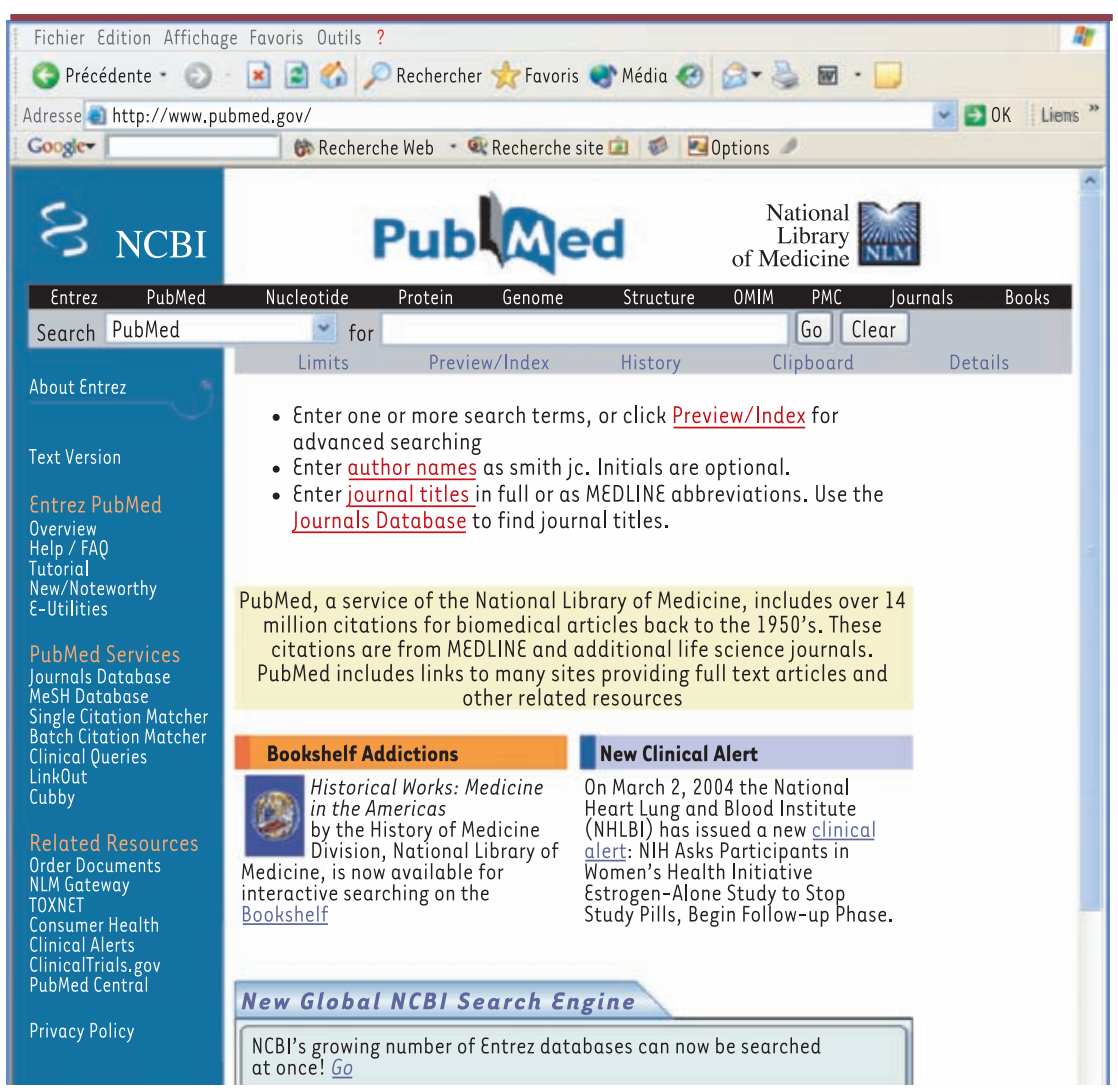

Figure 1. Page d'accueil de PubMed. Au dessus de la zone de saisie de l'équation de recherche, le bandeau noir horizontal donne accès à d'autres bases de données que PubMed. Le bandeau bleu sur le coté gauche permet d'accéder à des explications complémentaires sur PubMed (Entrez PubMed), à différentes fonctionnalités (PubMed services) ou bien encore à d'autres sources d'informations (Related Resources). définition des termes présents dans la liste pour guider l'utilisateur dans son choix. Lorsque celui-ci clique sur un de ces termes, il peut: (1) restreindre la portée de sa recherche en utilisant un ou plusieurs qualificatifs qui permettent de préciser le sens d'utilisation du terme MeSH choisi (exemples: toxicity, biosynthesis, history...); ainsi, I'utilisation du qualificatif «toxicity» pour le terme MeSH « genetically modified food » permet de retrouver seulement des articles qui abordent l'aspect «toxicité» des aliments génétiquement modifiés; (2) restreindre la recherche uniquement aux articles dont le sujet principal est le terme MeSH en question en cochant «Restrict search to major topic headings only»; (3) limiter sa recherche au terme MeSH choisi en excluant les notions plus spécifiques qui en découlent dans l'arborescence $\mathrm{MeSH}$, en cochant «Do not explode this term »; (4) utiliser le terme MeSH pour la recherche en cliquant sur le bouton «Send to», ce qui aboutit automatiquement à la requête correspondante dans la fenêtre d'interrogation de PubMed et permet d'accéder aux résultats de la recherche.

À noter que les termes MeSH anglo-saxons utilisés dans Medline PubMed peuvent être choisis en faisant appel au MeSH bilingue français-anglais de l'Inserm (http://disc.vjf.inserm.fr: 2010/basismesh/mesh.html).

\section{L'interface de recherche de Medline PubMed}

Si une bonne interrogation de Medline PubMed passe inévitablement par l'emploi du thésaurus MeSH, l'utilisation des champs de recherche et une exploitation adéquate des résultats obtenus sont tout aussi importantes pour mener à bien des recherches dans cette base.

\section{Formuler une équation de recherche} avec les champs de recherche

Chaque article référencé dans Medline PubMed est indexé avec de nombreux champs qui précisent, entre autres, le nom de l'auteur, le titre de l'article, le nom du périodique, la date de publication, l'adresse des auteurs... L'interrogation de ces champs permet d'effectuer des recherches plus spécifiques. II est par exemple possible de trouver des articles écrits par un auteur donné. Dans ce cas, la syntaxe utilisée doit être «makar ab $[A U]$ » pour trouver les articles écrits par AB Makar (AU est l'abréviation d'AUTHOR). 
II est également possible de repérer tous les articles publiés dans un périodique donné. La syntaxe est par exemple «Medecine sciences [J0]» pour retrouver les articles de Médecine Sciences indexés dans Medline PubMed (J0 est l'abréviation de JOURNAL). Cette procédure implique de connaître la syntaxe de chaque champ d'interrogation (AU pour auteur, J0 pour journal...), ce qui est loin d'être toujours le cas. Pour utiliser les champs d'interrogation sans en connaître la syntaxe exacte, il faut interroger l'interface «Preview/index», accessible depuis la barre de commandes située sous la fenêtre d'interrogation. II suffit alors de choisir le champ que l'on souhaite interroger dans le menu déroulant proposé (en bas à gauche), de saisir le terme à rechercher dans ce champ, et de cliquer sur « Preview » puis sur «Go» pour lancer la recherche. L'utilisation des champs de recherche est également très utile lorsqu'il n'existe pas de termes MeSH équivalents à l'un des mots de la requête (cela arrive parfois pour des notions extrêmement spécifiques ou très récentes). La recherche peut alors s'effectuer dans les mots du titre de l'article ou du résumé. Lorsqu'il est nécessaire d'interroger simultanément plusieurs champs de recherche, les termes peuvent être associés entre eux à l'aide des opérateurs booléens AND, OR et/ou NOT (saisis obligatoirement en majuscules ou choisis dans le menu déroulant de l'interface «Preview/index »).

\section{Exploitation des résultats}

Il est possible d'afficher les résultats selon plusieurs formats complémentaires les uns des autres. Par défaut, les résultats se présentent dans un format bibliographique traditionnel (auteurs, titre de l'article, nom du journal, pagination...). Si I'on clique sur un résultat, la référence choisie s'affiche selon un format plus complet présentant le résumé lorsqu'il existe. Le format «MEDLINE » permet de consulter tous les éléments constitutifs de l'indexation d'un article et doit être utilisé pour exporter des références vers un logiciel de gestion bibliographique de type «End Note» ou «Reference Manager».

Dans tous les cas, le menu déroulant «Sort» permet d'afficher les références par ordre chronologique (c'est l'affichage par défaut), par nom d'auteur, ou par périodique.

Le bouton «Send to» permet de créer un fichier texte (Send to text) utile pour copier-coller la référence vers un logiciel de traitement de texte ou envoyer le résultat par courrier électronique (Send to $\varepsilon$-mail), ce qui est très pratique lorsque l'on réalise des recherches bibliographiques en dehors de son laboratoire. II est également possible de stocker une série de références dans le «presse-papier» (Clipboard) de PubMed par la commande «Send to Clipboard ». Le presse-papier permet de visualiser, de regrouper, de sauvegarder et d'imprimer des références sur une seule page. Il est accessible depuis la barre de commandes située sous la fenêtre d'interrogation (Figure 1).

Il peut être intéressant d'utiliser la fonction «History », située sur la même barre de commande, qui récapitule les stratégies de recherche et le nombre de résultats obtenus.
L'accès au texte intégral des articles est parfois proposé gratuitement, mais il est le plus souvent conditionné à la souscription d'un abonnement payant.

\section{Recherche d'autres sources d'information}

Il est possible d'exploiter les résultats pour repérer d'autres sources d'information. À cet effet, la page de résultats de PubMed propose, en face de chaque notice bibliographique, un lien dénommé «Related Articles». Par la mise en jeu d'un algorithme comparant les mots du titre, du résumé et les mots du $\mathrm{MeSH}$, ce lien permet de retrouver les références proches d'un article. Les termes de la requête peuvent également être utilisés pour interroger un moteur de recherche comme «Google» ou «Alltheweb» et trouver des documents connexes disponibles en libre accès sur l'Internet. Dans ce cas, il convient d'être particulièrement vigilant quant à la validité des informations obtenues.

\section{Les autres fonctionnalités de Medline PubMed}

En complément de l'interface de recherche, Medline PubMed propose un certain nombre de fonctionnalités très utiles pour limiter l'étendue de ses recherches, compléter des références bibliographiques, systématiser des recherches, ou réaliser des revues de sommaires.

\section{Appliquer des limites à sa recherche}

Le menu «Limits», accessible depuis la barre de commandes située sous la fenêtre d'interrogation (Figure 1) permet de limiter les recherches à certains types de publications (essai clinique, éditorial, lettre aux éditeurs, revue de la littérature...), à une période de publication (de 30 jours à 10 ans), à une langue donnée (sept langues usuelles sont proposées), ou à un type d'étude (humaine ou animale, tranches d'âge étudiées).

\section{Compléter une référence partielle}

Medline PubMed permet, à travers l'interface «Single citation matcher », accessible depuis la barre latérale bleue, de retrouver un article dont on ne connaît pas la référence complète. Cette situation est assez fréquente, en particulier lorsqu'une diapositive présentée à l'occasion d'un congrès ne comporte que des références partielles (nom de l'auteur et année de publication). Cette interface permet de remplir les champs que l'on connaît (journal, date de publication, pagination, auteur, mots du titre) et de retrouver la référence complète. Lors de l'interrogation de cette interface, les dates doivent être indiquées selon le format étasunien, en entrant yyyy,yyy/MM ou yyyy/MM/J (par exemple: 1998, 1998/03 ou 1998/03/06 pour trouver un article paru le 6 mars 1998). Le nom de l'auteur et ses initiales doivent être placés entre guillemets (exemple: «makar $a b »)$. 
Systématiser ses recherches: «Cubby» et «Biomail»

Pour mettre en place une veille bibliographique, les requêtes pertinentes peuvent être sauvegardées dans l'interface «Cubby» de PubMed, accessible également depuis la barre latérale de couleur bleue, à gauche de l'écran (Figure 1). Les stratégies de recherche enregistrées peuvent être réutilisées à volonté pour retrouver les références nouvelles parues depuis la dernière connexion de l'utilisateur à ce service.

Même s'il ne fait partie intégrante de Medline PubMed, le service «Biomail» (http://biomail.sourceforge.net/biomail/ biomailfr.html) permet de recevoir gratuitement par courriel, à intervalles réguliers, les résultats de recherches effectuées dans Medline PubMed. II suffit pour cela, de s'inscrire à ce service, de saisir la (ou les) équation(s) de recherche correspondant aux recherches que l'on souhaite systématiser, telles qu'elles doivent être saisies dans l'interface Medline PubMed. Le plus simple est de réaliser un « copier-coller » à partir d'une requête fonctionnant correctement sur Medline PubMed. Dernière étape, attendre que les nouvelles références arrivent dans la boite aux lettres électronique. La fréquence d'envoi des résultats (de quotidienne à mensuelle) et le nombre maximal de références envoyées sont paramétrables.

\section{Rechercher un journal et faire une revue de sommaire}

L'interface «Journal Database» permet de savoir si un journal est indexé dans Medline PubMed, en saisissant simplement son nom dans la zone de saisie. Si c'est le cas, il est alors possible d'accéder à des informations complémentaires sur ce périodique (titre abrégé, nom de l'éditeur, langue de publication...) en cliquant sur le numéro situé après la mention «NLM ID». Cette interface permet également de réaliser une revue de sommaire en cliquant à droite de l'écran sur « Links » puis «PubMed». A noter qu'il est possible de systématiser ce type de recherche via «Biomail », pour recevoir dans sa boite aux lettres électronique les sommaires du ou des périodiques de son choix, dès la parution des nouveaux numéros.

\section{Intégrer la bibliographie à un traitement de texte ou à un logiciel de gestion bibliographique}

Les références bibliographiques identifiées avec Medline PubMed peuvent être intégrées dans un traitement de texte par une simple opération de «copier-coller» du fichier texte correspondant (bouton «Send to text»). II est également possible d'importer directement des références dans un logiciel de gestion de références bibliographiques personnelles comme «EndNote», «ProCite» ou « Reference Manager». Si les procédures diffèrent d'un logiciel à l'autre, il faut le plus souvent sauvegarder les résultats dans Medline PubMed au format « MEDLINE » et surtout ne pas oublier de choisir le filtre d'importation qui permet de lire les données provenant de Medline PubMed. Les avantages de ces logiciels de gestion bibliographiques sont nombreux: ils évitent notamment les épisodes fastidieux de saisies, sources d'erreurs de frappe qui se répercutent dans les bibliographies d'articles. Ils autorisent également le reformatage automatique des références sélectionnées en fonction des contraintes imposées par la revue à laquelle les articles sont destinés (plus de 1000 styles sont possibles avec EndNote).

\section{Conclusions}

Medline PubMed s'est imposée en quelques années comme le standard de la recherche bibliographique en biologie et médecine. La gratuité de l'accès à cette base, la qualité de son interface alliée à la possibilité d'utiliser le vocabulaire $\mathrm{MeSH}$, ainsi que les nombreuses fonctionnalités qu'elle propose (Journal Database, Cubby, History...) expliquent cette réussite. Malgré toutes ces qualités, il ne faut pas oublier que Medline est loin d'indexer tous les périodiques existant dans le domaine de la biologie et de la médecine. Cela signifie que la seule interrogation de PubMed est, à de rares exceptions près, loin d'être suffisante pour réaliser une bibliographie « exhaustive ». L'interrogation d'autres bases de données bibliographiques comme Embase ou Biosis, même si leur accès est plus contraignant car soumis à un abonnement payant, doit compléter celle de PubMed. II faut enfin souligner que la couverture de PubMed étant très majoritairement anglophone (plus de $85 \%$ des articles sont en anglais), les périodiques de langue française y sont sous-représentés.

Pour en savoir plus sur Medline PubMed, on peut consulter le site: http://www.ccr.jussieu.fr/urfist/biolo/bioguide2/medline/medline.htm. $\Delta$

\section{SUMMARY}

\section{A tutorial for Medline PubMed}

To be informed about the last publications recently published or to produce a bibliography in a given thematic field is essential for researchers in the biomedical field. If the use of Internet information searching tools such as «Google» or «Alltheweb» makes possible to discover a great part of the grey literature, bibliographic databases like Embase, Current Contents, Biosis or Medline via PubMed are essential tools to locate scientific articles. Among these bibliographic databases, Medline PubMed, thanks to its free access, is the most used. However, a correct utilization of the various functionalities proposed (thesaurus MeSH, systematization of bibliographic searches...), and consequently the quality of bibliographic researches carried out in this database, requires to master elementary knowledge which are exposed in this article. $\Delta$

\section{RéFÉRENCES}

1. Boudry C. Typologie et mode de fonctionnement des outils de recherche d'information sur Internet en biologie/médecine. Med Sci (Paris) 2002; 18: 616-22.

2. Auriol P, Bernardin G, Boudry C, et al. Fiabilité de l'information médicale sur le Net: «Les couleurs du web». Web Med (Web guide médical) 2002; 4: 20-1.

3. Wood FB, Lyon B, Schell MB, et al. Public library consumer health information pilot project: Results of a national library of medicine evaluation. Bull Med Libr Assoc 2000; 88: 314-22.

\section{TIRÉS À PART}

C. Boudry 